\title{
Late Presenting Pediatric Radial Neck Fracture: A Case Report and Review of the Literature
}

\author{
Kaya Turan ${ }^{1}$, Cenk Köroğlu ${ }^{2}$, Haluk Çabuk ${ }^{1}$ \\ Learning Point of the Article:
}

Even the diagnosis of displaced pediatric radial neck fractures is delayed excellent functional and radiologic outcomes are obtainable by open reduction.

\section{Abstract}

Introduction: A pediatric radial neck fracture, when the diagnosis is delayed, is still a challenging problem for the treating orthopedic surgeon. We report a pediatric patient with late presenting radial neck fracture and results of open reduction and fixation with Kirschner wires.

Case Report: A 13-year-old right-handed girl fell on an out-stretched right arm after being tackled during playing basketball and had a radial neck fracture. However, the diagnosis of her fracture was delayed for 3 weeks. At the first radiologic examination, the anteroposterior and lateral radiographs were showing over 80 degrees of angulation at the radial neck and subluxation of the radiocapitellar joint. We tried to obtain a closed reduction, but we could not succeed. Then, we performed open reduction while preserving medial periosteal continuity and vascular supply of the radial head by meticulous surgical dissection. Sixth-month radiography control and clinical examination confirmed the complete healing of the fracture without any epiphyseal injury. Painless full range of motion without any restriction of pronation and supination was achieved. The patient and her parents were satisfied with the outcome.

Conclusions: Even if the diagnosis of pediatric radial fractures is delayed if we can preserve medial periosteal continuity and vascularity of the radial head with open reduction, satisfactory results are obtainable.

Keywords: Pediatric, radial neck fracture, open reduction, late diagnosis.

\section{Introduction}

Pediatric radial neck fractures are common traumatic injuries that usually affect the proximal metaphysis of radius. The ossification center of the radial head may be bipartite and appears between 3 and 5 years of age. The radial head fuses with the metaphysis at 16-18 years. Pediatric neck fractures are commonly seen in children 9-10 years of age. The classification and treatment depend on angulation. Metaizeau described the commonly used and accepted classification is a modified classification which was primarily designated by Judet in the 1980s. Type 1 is non-displaced, type 2 has $<30$-degree angulation, type 3 has between 30 and 60 degrees, and type 4 has two sub-types. Type $4 \mathrm{a}$ has between 60 and 80 degrees and type $4 \mathrm{~b}$ has over 80 degrees of angulation. Another classification described by Chambers is used, less common, and based on the primary displaced part of the proximal radius.

Non-displaced fractures may be difficult to visualize on standard anteroposterior and lateral radiographs. A fat pad sign may be an indicator of an intra-articular fracture. The fleck sign described by Zhu may be the only sign of a completely displaced radial neck fracture and is diagnostic for pediatric patients who have no secondary ossification of the radial head [1]. Treatment for type 1, <30 degrees of angulation, involves immobilization alone, for over 30 degrees of angulation and $2 \mathrm{~mm}$ of translation closed reduction should be considered. Over 30 degrees of residual angulation, 3-4 $\mathrm{mm}$ of translation, and $<45$ degrees range of motion in pronation and supination following closed reduction, which are indications for percutaneous fixation.

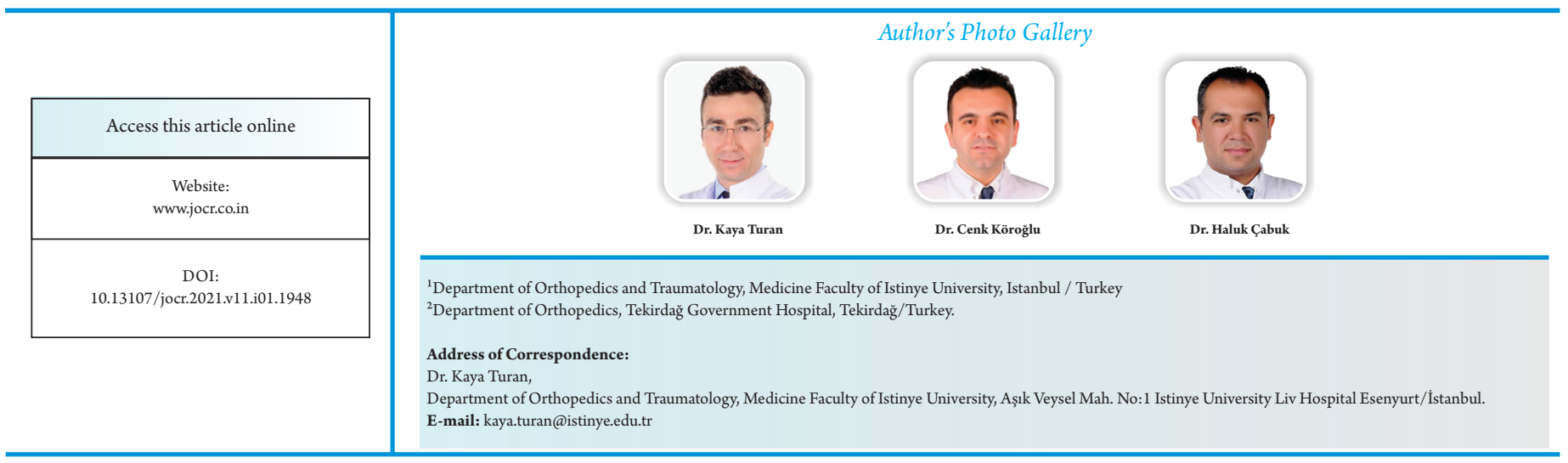

Journal of Orthopaedic Case Reports | pISSN 2250-0685 | eISSN 2321-3817 | Available on www.jocr.co.in | doi:10.13107/jocr.2021.v11.i01.1948 This is an Open Access article distributed under the terms of the Creative Commons Attribution Non-Commercial License (http://creativecommons.org/licenses/by-nc/3.0) which permits unrestricted non-commercial use, distribution, and reproduction in any medium, provided the original work is properly cited. 


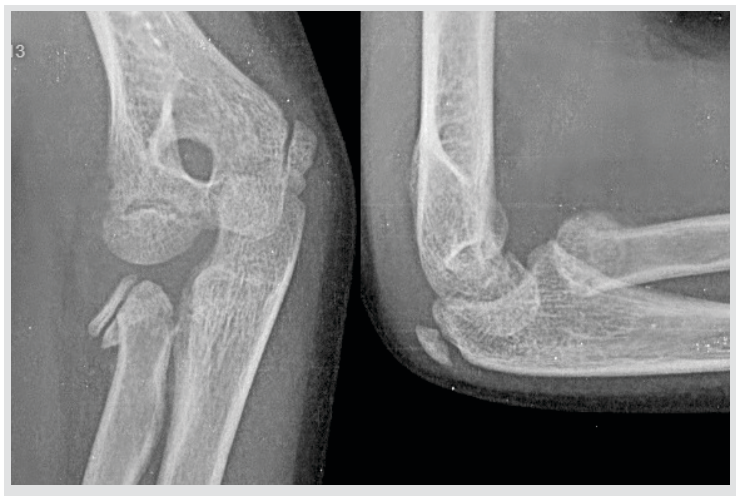

Figure 1: Pre-operative AP-lateral views.

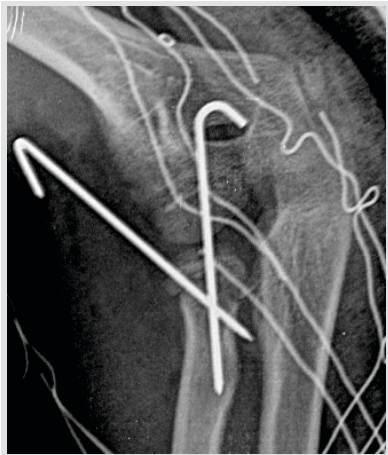

Figure 2: Post-operative early radiography.

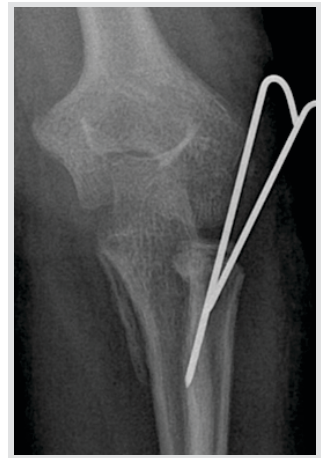

Figure 3: Post-operative 3rdweek radiography

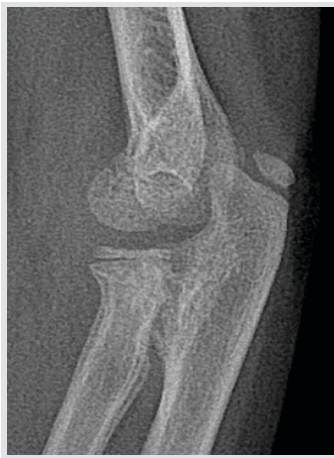

Figure 4: Post-operative 6th-week radiography.
Percutaneous fixation may be done by retrograde intramedullary elastic nailing or directly at the fracture site. Open reduction is reserved for fractures that cannot be adequately reduced to $<45$ degrees of angulation with closed or percutaneous methods.

\section{Case Report}

A 13-year-old right-handed girl fell on an out-stretched right arm after being tackled while playing basketball. The patient did not feel pain in the early stages. As the swelling of the elbow increased, the patient admitted to a state hospital's emergency service. A dorsal splint was applied as a first intervention and suggested for an orthopedic consultation. Two days later, an orthopedic surgeon examined the patient and made a closed reduction in the office. The surgeon ordered control radiography, but the patient left the hospital without control. The patient admitted 3 weeks later, and we removed the splint. The anteroposterior and lateral radiographs showed over 80 degrees of angulation at the radial neck and luxation of the radiocapitellar joint (Fig. 1). At this point, we decided on the surgical intervention. We attempted closed reduction maneuvers in the operation room, but we could not achieve an acceptable reduction. We corrected the radial head by a lateral approach with an osteotomy from the metaphyseal region while

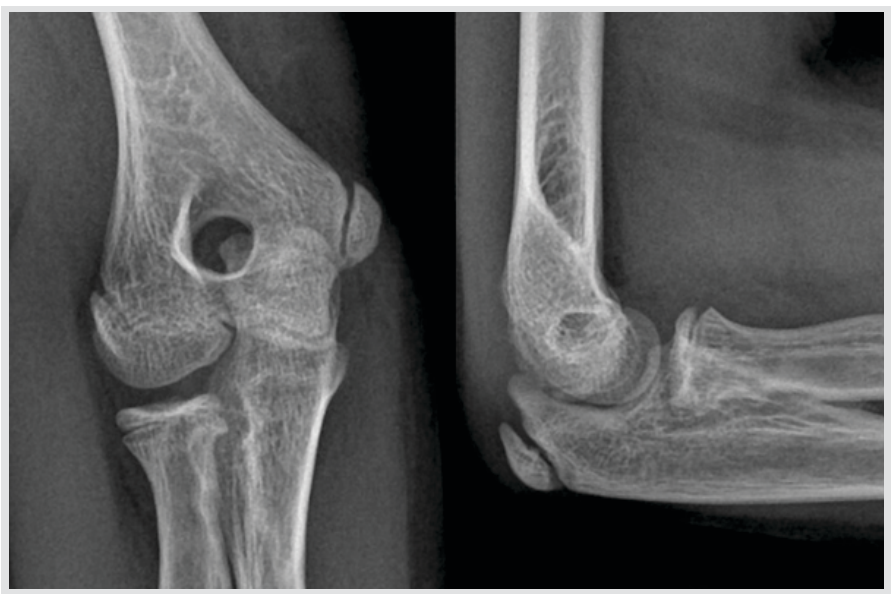

Figure 5: Post-operative 2 years radiography preserving medial periosteal continuity. We secured the reduction by two trans-epiphyseal percutaneous $1.7 \mathrm{~mm}$ Kirschner wires. We applied a dorsal splint to hold the elbow at 90 degrees of flexion (Fig. 2). The splint discontinued in the $3 \mathrm{rd}$ week post-operatively and started a gentle range of motion exercises for the elbow and wrist (Fig. 3). Kirschner wires were removed in the 1 st month at the office, then started physiotherapy to stretch and strengthen (Fig. 4). Complete healing of the fracture with the alignment of the radial neck and radiocapitellar joint with no epiphyseal injury was provided by post-operative 6th-month follow-up. Painless full range of motion without any restriction on pronation and supination was achieved (Fig. 5, 6).

\section{Discussion}

Displaced radial neck fractures remain a challenging problem for treating orthopedic surgeons, significantly when the diagnosis is delayed. Immobilization alone is adequate for minimally displaced fractures. However, fractures with over 30 degrees of angulation may require closed reduction with or without percutaneous fixation. If closed and anatomical reduction cannot be achieved, open reduction should be considered. The aim of the treatment is restoring the radial length and anatomical relationship of the radiocapitellar joint.

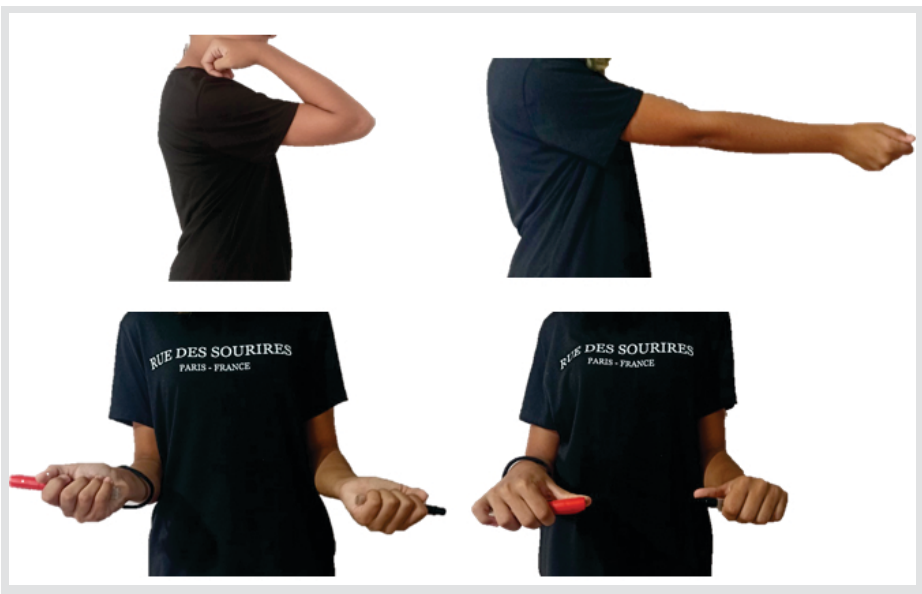

Figure 6: Clinical images of the final outcome. 
Various techniques have been described in the literature for this purpose. Metaizeau et al. described the closed reduction with retrograde intramedullary nailing to protect vascularization of the radial head and proximal radial epiphysis, thus avoiding epiphysiolysis [2]. In the literature, there are several publications about the results of early surgical interventions. Tarallo et al. compared intramedullary versus percutaneous pinning techniques and showed superior results for intramedullary nailing, lower complication rates, and a higher range of motion values [3]. In acute settings, closed reduction and intramedullary elastic nailing can be aided with lever arm manipulation by percutaneous Kirschner wire [4]. Correspondingly, another technique described by Dawson and Inostroza is reducing radial head angulation with arthroscopy and fixing the radial head percutaneously [5]. They achieved good to excellent results and normal bone morphology and function. Nevertheless, these techniques are described for early surgical interventions. When we look up the literature for late presenting radial neck fractures, we seemed only a few case reports. Majed and Baco reported a case report of closed reduction and intramedullary elastic nailing 5 days from the initial trauma [6]. They concluded that fixation with intramedullary wires is safe and effective even when the presentation is delayed up to 1 week after injury. Papageorgiou presented a patient who had a delayed open reduction and transarticular Kirschner wire fixation at 120th day of injury showed no sign of neither avascular necrosis nor joint stiffness [7]. They concluded that maintaining the viability of the radial head is due to preserving the medial attachment of the radial head and limiting the hardware usage. Gutierrez-De La Iglesia et al. reviewed that 51 patients that have Judet Grade 3 and Grade 4 fractures have shown that there are no significant differences in complications or final functional results between closed and open reduction. Only the initial fracture displacement may be an independent predictor of functional outcome [8]. In our case report, we applied the surgical treatment with an open approach, at the end of the $3 \mathrm{rd}$ week from the initial trauma.

\section{Clinical Message}

We believe that protecting the medial region of the radial neck has vital importance to prevent avascular necrosis, intraarticular calcification, joint stiffness, as well as decreasing carrying angle of the elbow. Even the presentation or diagnosis of pediatric radial neck fractures is delayed if the open reduction is achieved while preserving radial head vascular supply by meticulous surgical dissection, good functional and radiologic outcomes are obtainable.

\section{References}

1. Zhu AF, Sponseller PD. An undiagnosed fracture of the radial neck in a young child: The radial neck fleck sign: A case report.JBJS Case Connect 2013;3:e97.

2. Metaizeau JP, Lascombes P, Lemelle JL, Finlayson D, Prevot J. Reduction and fixation of displaced radial neck fractures by closed intramedullary pinning. J Pediatr Orthop 1993;13:355-60.

3. Tarallo L, Mugnai R, Fiacchi F, Capra F, Catani F. Management of displaced radial neck fractures in children: Percutaneous pinning vs. elastic stable intramedullary nailing.J Orthop Traumatol 2013;14:291-7.

4. Koca K, Erdem Y, Neyişci Ç, Erşen Ö. Intramedullary elastic nailing of the displaced radial neck fractures in children.
Acta Orthop Traumatol Turc 2017;51:451-4.

5. Dawson FA, Inostroza F. Arthroscopic reduction and percutaneous fixation of a radial neck fracture in a child. Arthroscopy 2004;20:90-3.

6. Majed A, Baco AM. Late diagnosis and treatment of a paediatric radial neck fracture. Inj Extra 2006;37:322-4.

7. Papageorgiou. Treatment of A Late Presenting Displaced Radial Neck Fracture in A 10 Years-old Girl. J Med Cases 2011

8. Gutiérrez-De La Iglesia D, Pérez-López LM, CabreraGonzález M, Knörr-Giménez J. Surgical techniques for displaced radial neck fractures: Predictive factors of functional results.J Pediatr Orthop 2017;37:159-65
Conflict of Interest: Nil

Source of Support: Nil

Consent: The authors confirm that informed consent was obtained

from the patient for publication of this case report

\section{How to Cite this Article}

Turan K, Köroğlu C, Çabuk H. Late Presenting Pediatric Radial Neck Fracture: A Case Report and Review of the Literature.. Journal of Orthopaedic Case Reports 2021 January; 11(1):20-22. 